\title{
Epidémiologie de l'éjaculation prématurée et de son cumul avec la dysfonction érectile
}

\author{
A. BÉJIN \\ Directeur de Recherche au CNRS*
}

\section{RÉSUMÉ}

L'éjaculation prématurée (EP) est la dysfonction sexuelle masculine la plus répandue. D'après l'enquête du groupe ACSF dont j'ai fait partie (septembre 1991-février $1992 ; n=$ 20055), 5\% des hommes sexuellement actifs de 18 à 69 ans en France ont souvent des éjaculations ante portas (avant la pénétration) et $10 \%$ souvent des éjaculations prématurées non ante portas (pendant ou après la pénétration). Ainsi, $11 \%$ des hommes de 18 à 69 ans souffrent d'EP “ tout court" (i.e. ante portas et/ou non ante portas) souvent, et $65 \%$ en souffrent au moins occasionnellement. Les chiffres correspondants pour la dysfonction érectile (DE) sont de $7 \%$ et $47 \%$. Ces données font l'objet de comparaisons dans le temps (avec les résultats d'une enquête française de 1970) et dans l'espace (EU et Finlande). Est particulièrement étudié, dans l'article, le cumul de l'EP et de la DE : $26 \%$ des 18-24 ans n'ont eu ni DE ni EP, 11\% ont souffert de DE mais pas d'EP, 41\% d'EP mais pas de DE et $22 \%$ ont déclaré avoir souffert au moins occasionnellement à la fois d'EP et de DE. Les chiffres correspondants pour les 60-69 ans sont de 4\%, $27 \%, 28 \%$ et $41 \%$. Je propose, dans l'article, quelques hypothèses susceptibles d'expliquer l'évolution des ces prévalences. Je propose également une typologie des EP.

Mots-clés : Sexualité, épidémiologie, éjaculation prématurée, dysfonction érectile, désir sexuel, France, comparaisons internationales.
L'éjaculation prématurée (EP) est la dysfonction sexuelle masculine la plus répandue. C'est, en même temps, une dysfonction difficile à traiter. Les thérapies sexologiques comportementalistes à la Masters-Johnson, pourtant nettement plus efficaces que la psychanalyse par exemple [3], se solderaient, aux EU notamment, par des échecs (i.e. des " rechutes" moins de 3 ans après la fin de la cure) dans au moins $75 \%$ des cas [12]. Pourtant cette dysfonction n'a fait l'objet d'aucun travail épidémiologique approfondi, fondé sur un échantillon représentatif de la population générale.

Il a fallu attendre les enquêtes nationales des années 90 en France, en Finlande et aux EU pour disposer de données relativement fiables $[1,4,5,9,10,15]$. Mais l'enquête américaine n'a pris en considération que l'EP persistante. Et l'enquête finlandaise s'intéresse aux dysfonctions des seuls hommes ayant une partenaire stable et ne propose, à leur sujet, que des commentaires succincts. L'enquête française ACSF, à laquelle j'ai participé, est donc la seule qui nous renseigne sur les EP persistantes, intermittentes ou occasionnelles de l'ensemble des hommes adultes sexuellement actifs.

Après avoir envisagé les différentes définitions possibles de l'EP, j'examinerai la thèse selon

\footnotetext{
* (UMR CNRS 8596 - Centre Roland Mousnier Histoire et Civilisation - Université de Paris - Sorbonne - Paris IV) 9, rue de Guise 02140 Vervins, France.
} 
laquelle l'EP ne constituerait pas une dysfonction sexuelle. Je comparerai, ensuite, les prévalences de l'EP aux prévalences d'autres dysfonctions puis à celles fournies par d'autres enquêtes. J'examinerai, enfin, comment, en fonction de leur âge, les hommes cumulent plus ou moins, ou ne cumulent pas, l'EP et la dysfonction érectile (DE). Je proposerai quelques hypothèses susceptibles d'expliquer l'évolution de ces prévalences de cumul et de non-cumul de l'EP et de la DE.

\section{MATERIEL ET METHODES}

L'importance prise par l'épidémie de sida dans les années 80 a entraîné le lancement au début des années 90, d'enquêtes quantitatives nationales de grande ampleur sur les comportements sexuels, notamment en France $[1,4,5,15]$, en Finlande [9] et aux Etats-Unis [10].

L'enquête à laquelle je vais principalement me référer est l'enquête du groupe ACSF ("Analyse des Comportements Sexuels en France"). Elle a été réalisée sous le patronage et avec le soutien de l'Agence Nationale de Recherches sur le Sida (ANRS), de la Direction Générale de la Santé (DGS), du Comité Français d'Education pour la Santé (CFES) et de l'Agence Française de Lutte contre le Sida (AFLS).

Elle a été menée par une équipe formée d'épidémiologistes, de sociologues, de démographes, de psychosociologues, de psychologues, de psychanalystes, d'économistes appartenant à des institutions variées : INSERM, CNRS, INED, Universités de l'Ile-de-France. Elle a été coordonnée par A.Spira et N.Bajos.

Cette enquête a visé surtout à mieux comprendre les transformations des comportements sexuels et les logiques de protection face à l'épidémie de sida : 20.055 personnes de 18 à 69 ans, choisies aléatoirement, ont été, à cet effet, interrogées par téléphone de septembre 1991 à février 1992.

Les données dont je vais faire état se rapportent à une partie seulement de cet échantillon, qui a répondu à ce que nous avons appelé les "questionnaires longs ". Les données ayant trait aux domaines dont j'étais le responsable dans le groupe ACSF - les dysfonctions sexuelles, mais également des domaines non pris en considération ici : masturbation, qualité des orgasmes, attitudes vis-à-vis des humeurs corporelles, sentiments associés aux rapports sexuels et à la masturbation, fantasmes sexuels - ces données ont été établies à partir d'un sous-échantillon de 1339 hommes et 1139 femmes.

Les autres enquêtes quantitatives portant sur des échantillons représentatifs de la population générale auxquelles je me référerai sont les suivantes :

- l'enquête de P.Simon et de ses collègues [14] : 2625 personnes de 20 ans et plus, choisies selon la méthode des quotas, ont répondu à des questionnaires oraux (en face à face) et écrits (auto-administrés) de juin à septembre 1970.

- l'enquête finlandaise [9] : 2250 personnes de 18 à 74 ans, choisies aléatoirement, ont répondu à des questionnaires oraux (en face à face) et écrits (auto-administrés) de novembre 1991 à février 1992.

- enfin, l'enquête américaine [10], laquelle a porté sur 3432 personnes de 18 à 59 ans, choisies aléatoirement, et qui ont répondu à des questionnaires oraux (en face à face) et écrits (auto-administrés) de février à septembre 1992.

Quelles questions avons-nous posées dans l'enquête ACSF, pour identifier l'EP ? On sait $[12,16]$ qu'il existe de nombreuses définitions possibles de cette dysfonction. On peut mettre l'accent, notamment, sur la durée de la pénétration, le nombre de mouvements de va-etvient du pénis dans le vagin avant l'éjaculation, le non-contrôle du réflexe éjaculatoire, l'incapacité d'éjaculer au moment désiré, ou encore l'incapacité de satisfaire la partenaire.

Nous avons choisi de poser deux questions afin de pouvoir distinguer l'éjaculation " ante portas" et l'éjaculation " non ante portas" (ce qui n'est pas fait dans les autres enquêtes). D'autre part, figure, dans chacune de ces deux questions, la locution " trop rapidement ", laquelle peut traduire une incapacité de 
contrôler son réflexe éjaculatoire, ou d'éjaculer au moment désiré, ou bien encore une incapacité de satisfaire la partenaire.

Dans l'enquête ACSF, sept questions portent sur les dysfonctions masculines [5]. Je traiterai ici essentiellement de l'EP et de ses rapports avec la DE. J'évoquerai les troubles du désir sexuel au cours de la comparaison France Etats-Unis. Les données concernant l'éjaculation retardée, l'absence d'éjaculation et l'absence d'orgasme ne seront pas examinées ici.

Les deux questions sur l'EP et les questions sur la $\mathrm{DE}$ et les problèmes de désir étaient les suivantes (réponses possibles : souvent/parfois/assez rarement/jamais/non réponse).

\section{“Avez-vous déjà présenté les signes suivants :}

Q 338 A : “ Vous n'avez pas du tout d'érection (impuissance)"

Q 338 B : “ Vous éjaculez trop rapidement avant même de pénétrer votre partenaire"

Q 338 C : " Vous éjaculez trop rapidement au moment de la pénétration ou peu après elle"

Q 338 F : "Vous avez une absence ou insuffisance de désir sexuel ".

Ces questions figuraient vers la fin du questionnaire, après les questions sur la faculté de parvenir à l'orgasme selon les différentes pratiques sexuelles, et avant les questions sur les fantasmes sexuels.

Les réponses à ce type de questions, on le sait, se réfêrent généralement à ce qui a été éprouvé au cours de la période récente.

La formulation de ces questions peut sembler un peu trop tranchée. Mais rappelons qu'il s'agissait d'une enquête téléphonique et qu'il fallait, à la fois, être explicite et concis.

Les dysfonctions déclarées comme se produisant " souvent", " parfois ", " assez rarement" seront ici qualifiées, respectivement, par les adjectifs " persistantes ", " intermittentes ", et "occasionnelles". "Au moins occasionnellement " signifiera : " soit souvent, soit parfois, soit assez rarement ". Pour qualifier les dys- fonctions se produisant " souvent", je n'utiliserai pas les adjectifs " graves" ou "sévères " car certaines personnes peuvent se sentir "gravement "affectées par des dysfonctions qu'elles déclarent pourtant "intermittentes", alors que d'autres ne se sentent pas " gravement " affectées par des dysfonctions se produisant, de leur aveu même, fréquemment.

Après avoir distingué l'éjaculation ante portas (Q $338 \mathrm{~B})$ et l'éjaculation non ante portas ( $Q$ $338 \mathrm{C}$ ), je regrouperai les réponses de la façon suivante (après avoir exclu les non réponses, lesquelles sont inférieures à $2 \%$ ) :

EP “ souvent” : l'une des deux, ou les deux, "souvent"

EP “ parfois" : l'une des deux, ou les deux, "parfois" (mais pas " souvent")

EP “ assez rarement" : l'une des deux, ou les deux, " assez rarement" (mais ni " souvent", ni " parfois ")

EP “ jamais" : les deux "jamais"

Signalons, pour terminer, que notre questionnaire ne nous permet pas d'établir si la dysfonction est “ primaire ", c'est-à-dire présente dès le premier rapport sexuel, ou " secondaire ", apparue plus tard.

\section{L'EP EST-ELLE UNE DYSFONCTION SEXUELLE ?}

Kinsey $[7,8]$ remarquait que, dans de nombreuses espèces de mammifères, et notamment de primates, l'éjaculation suit presque instantanément l'intromission. Il notait que les hommes peuvent parvenir à l'orgasme très rapidement par la masturbation, comme certaines femmes d'ailleurs. Il en tirait la conclusion qu'en règle générale l'EP des Américains n'est pas une dysfonction sexuelle, mais qu'elle résulte de l'inhibition ou de la faible libido de nombreuses Américaines... L'idée que l'homme devrait se retenir jusqu'à l'orgasme de sa partenaire, laissait-il entendre, serait une invention arbitraire récente due à certains cliniciens. Le raisonnement est spécieux, et la dernière affirmation manifestement fausse. En effet, la valorisation du contrôle, par l'homme, 
de son éjaculation pour mieux permettre à la femme d'obtenir elle aussi du plaisir n'est pas un phénomène récent. Il y a deux mille ans, Ovide écrivait [13] : " il ne faut pas hâter le terme de la volupté, mais y arriver insensiblement après des retards qui la diffèrent. (...). Le but, atteignez-le en même temps ". Au IVème siècle de notre ère, Vâtsyâyana [17] remarque “ que l'homme dont l'activité dure longtemps plaît au femmes ; par contre elles se plaignent des hommes qui jouissent rapidement ". Quinze siècles plus tard, en 1864 exactement, un auteur inconnu se désignant soi-mêmc en ces termes "Un octogénaire : J'ai vécu, je voudrais être utile à ceux qui ont vécu " publie une parodie de l'Art poétique de Boileau, dont j'extrais ces quelques vers [2] :

“Quel que soit le jupon sous lequel on s'escrime,

Bander est un devoir, et mollir est un crime. (...)

Quoiqu'un foutre brûlant vous excite et vous presse,

$\mathrm{Ne}$ vous piquez jamais d'une folle vitesse.

Pour un con affamé c'est un triste présent

Qu'un vit qui bande, enconne et décharge à l'instant".

Cette valorisation de la maîtrise, par l'homme, de son éjaculation, de façon à favoriser le plaisir de la femme, semble s'être accentuée au XXème siècle. L'enquête de P.Simon et al. [14], comme l'enquête $\mathrm{ACSF}$, montrent qu'une nette majorité des hommes comme des femmes considèrent l'orgasme partagé, voire l'orgasme simultané [3], des partenaires comme hautement souhaitable, même si la norme de l'orgasme simultané semble être devenue moins impérative depuis les années 70 [4]. Compte tenu de cette attente normative, il est difficile de prétendre qu'une EP persistante (voir intermittente) mal tolérée par l'homme et par sa partenaire ne constitue pas une dysfonction sexuelle. Toutefois, on peut concéder à Kinsey que l'EP intermittente ou occasionnelle est " normale" statistiquement dans la mesure où plus d'un homme sur deux y est, de temps à autre, confronté, comme nous allons maintenant le voir (Tableau 1).

\section{PREVALENCES ET COMPARAISONS}

On le voit dans le tableau 1 , les prévalences de l'EP ante portas et de l'EP non ante portas tendent à augmenter avec l'âge. Ainsi, contrairement à ce qui est parfois affirmé, la prévalence de ces deux formes d'EP n'est pas plus élevée chez les jeunes (je reviendrai sur ce point).

La progression des prévalences des deux formes d'EP en fonction de l'âge est moins régulière que celle des prévalences de la $\mathrm{DE}$ mais, dans l'ensemble, plus régulière que celle des prévalences des troubles du désir, lesquelles augmentent surtout après 45 ans.

Comment les deux formes d'EP persistante se cumulent-elles ? Chez les hommes sexuellement actifs de 18 à 69 ans en France, 85\% de ceux qui souffrent d'EP ante portas persistante sont également affectés d'EP non ante portas persistante et $40 \%$ de ceux qui souffrent d'EP non ante portas persistante sont également affectés d'EP ante portas persistante. Ainsi, $11 \%$ des hommes de 18 à 69 ans souffrent d'EP persistante, "tout court" (i.e. ante portas et/ou non ante portas) : 6\% souffrent seulement d'EP non ante portas persistante, $4 \%$ cumulent les deux et 1\% des 18-69 ans sont affectés d'EP ante portas persistante mais pas d'EP non ante portas persistante. Ce pourcentage de $1 \%$ (les cas considérés généralement comme les plus “graves ") représente, ainsi, un peu moins de 10\% de l'ensemble des cas d'EP persistante : c'est exactement le même pourcentage que celui avancé par Masters, Johnson et Kolodny [11] pour les Etats-Unis.

Voyons, maintenant, comment se cumulent " au moins occasionnellement" les deux formes d'EP : 88\% des 18-69 ans affectés au moins occasionnellement d'EP ante portas la cumulent avec de l'EP non ante portas ; $60 \%$ des 18 69 ans affectés au moins occasionnellement d'EP non ante portas la cumulent avec de l'EP ante portas. Ainsi, 65\% des hommes de 18 à 69 ans souffrent au moins occasionnellement d'EP : $24 \%$ seulement d'EP non ante portas, $5 \%$ seulement d'EP ante portas et, enfin, $36 \%$ cumulent les deux.

Toujours chez les hommes sexuellement actifs de 18 à 69 ans, les prévalences des autres dysfonctions, dans l'enquête ACSF, sont, par ordre 
Tableau 1: Distribution de quatre dysfonctions chez les hommes adultes vivant en France et ayant déjà eu, à la date de l'enquête ACSF (septembre 1991-février 1992), au moins un rapport sexuel, selon l'âge et la fréquence déclarée de la dysfonction (en \%).

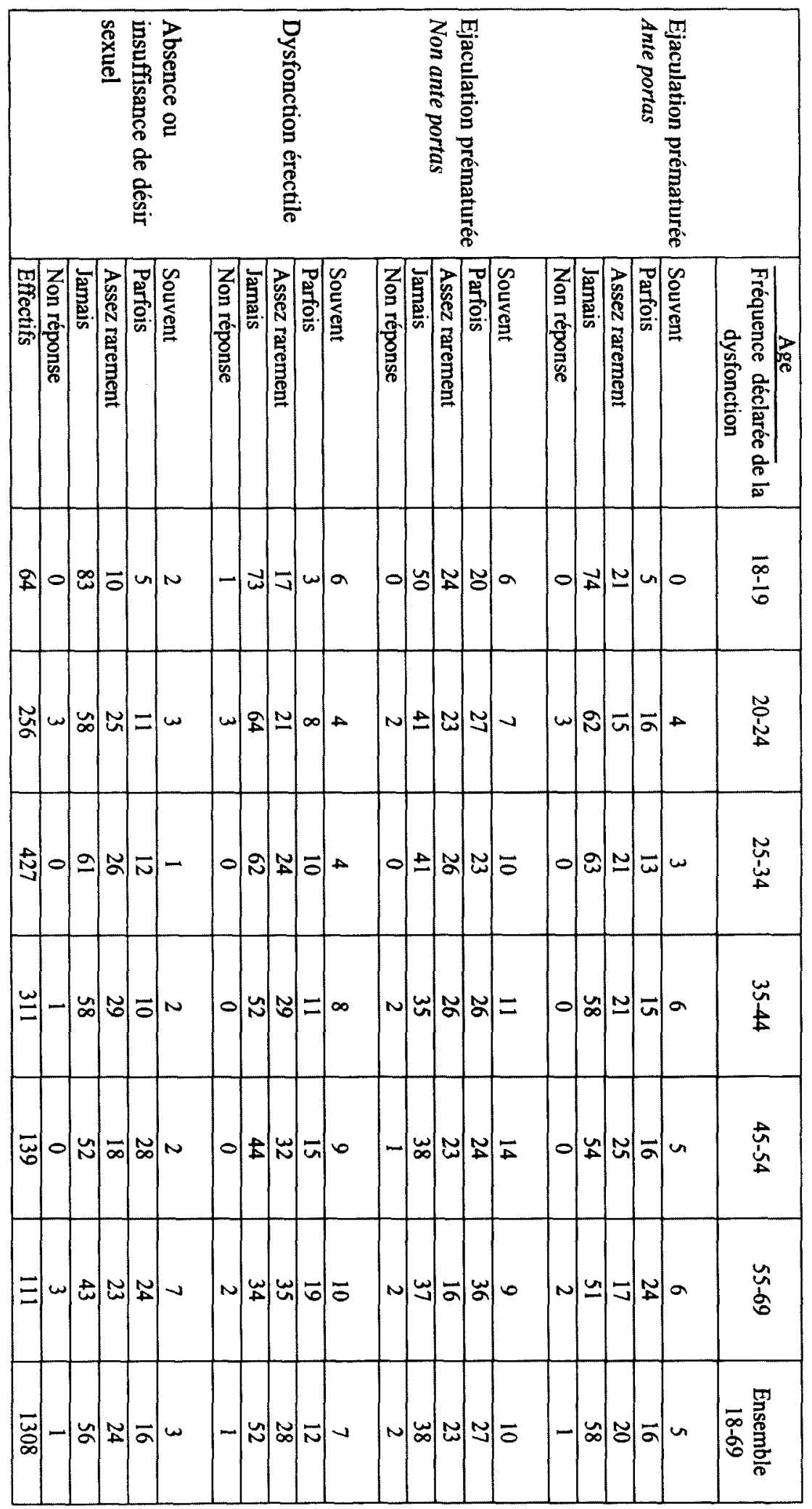


décroissant, les suivantes :

-éjaculation retardée : $49 \%$ (dont $4 \%$ souvent)

-DE : $47 \%$ ( $7 \%$ souvent)

-absence ou insuffisance de désir : 43\% (3\% souvent)

-anorgasmie : $34 \%$ (7\% souvent)

-anéjaculation : $24 \%$ ( $2 \%$ souvent)

Et, chez les femmes sexuellement actives de 18 à 69 ans :

- absence ou insuffisance de désir : 64\% (8\% souvent)

-anorgasmie (ou "frigidité ") :56\% (11\% souvent)

-dyspareunie (rapports sexuels douloureux ) : $50 \%$ ( $5 \%$ souvent)

Signalons que ces chiffres représenteraient actuellement, en France, autour de 1,5 million d'hommes adultes (de 18 à 69 ans) souffrant de DE persistante et autour de 2,4 millions affectés d'EP persistante.

Comparons maintenant les prévalences établies dans les différentes enquêtes.

Aux Etats-Unis [10], chez les hommes de 18 à 59 ans ayant eu au moins un rapport sexuel dans les 12 mois précédents, $28,5 \%$ ont déclaré être affectés d'EP persistante (l'enquête américaine n'a malheureusement pas pris en considération l'EP intermittente ou occasionnelle).

C'est nettement plus que chez les Français de $18-59$ ans $(11,4 \%)$.

La prévalence correspondante en Finlande [9] semble se situer autour de $20 \%$ (la comparaison est plus difficile avec ce pays).

L'écart France -Etats-Unis de prévalence de l'EP persistante (France : 11,4\% ; Etats-Unis : $28,5 \%$ ) est ainsi plus important que l'écart de prévalence de la DE persistante (France : $6,6 \%$; Etats-Unis : $10,4 \%$ ) et comparable, mais pour les hommes seulement, à l'écart de prévalence des troubles persistants du désir (chez les hommes : 1,7\% en France contre 15,8\% aux Etats-Unis ; chez les femmes : $7 \%$ en France contre $33,4 \%$ aux Etats-Unis). On notera, en passant, que les différences de culture érotique entre pays influent plus sur les prévalences de
l'EP et des troubles du désir persistants que sur celle de la DE persistante.

Comment expliquer cet écart important, méconnu, jamais encore établi statistiquement ? Les Français se seraient-ils montrés plus indulgents envers eux-mêmes que les Américains et auraient-ils comparativement sous-déclaré leur EP persistante? Cette explication doit être écartée pour les raisons suivantes.

Les difficultés sexuelles auxquelles les femmes sont sujettes peuvent parfois résulter des dysfonctions de leurs partenaires masculins. Supposons que l'EP soit la seule cause de l'anorgasmie féminine persistante (ce qui est une hypothèse bien hardie). Quelle est la prévalence de cette anorgasmie persistante déclarée par les femmes : $11 \%$, c'est-à-dire exactement le pourcentage d'EP persistante déclarée par les hommes.

Soyons plus exigeants encore. L'EP est un problème de synchronisation des orgasmes masculin et féminin dans le coït vaginal. Or, quel est le pourcentage des femmes qui déclarent parvenir " assez rarement " ou “ jamais ” à l'orgasme simultané ? Il est de $16 \%$ ( $8 \%$ chez les hommes). Cela signifierait que la prévalence d'EP persistante tournerait, au pire, autour de $16 \%$. Mais, même si l'on suppose que toute la responsabilité de cette situation incombe aux seuls hommes, l'EP ne peut être la seule dysfonction à mettre en cause : la DE persistante y contribue aussi. Or, quel est le pourcentage d'hommes qui souffrent " souvent" d'EP et /ou de DE (cf. tableau 2) : il est précisément de $16 \%$.

Ajoutons que les prévalences de l'EP persistante et de l'anorgasmie féminine persistante sont assez proches l'une de l'autre aux EtatsUnis (respectivement $28,5 \%$ et $24,1 \%$ ) tout comme en France ( $11 \%$ et $10,5 \%)$.

Bref, il faut convenir que, dans l'enquête ACSF, les hommes n'ont pas (ou n'ont que peu) sous-déclaré leur EP persistante. Donc la différence de prévalence de l'EP persistante entre la France et les Etats-Unis est un fait réel.

Il faudrait, pour tenter d'expliquer cet écart, mettre en lumière les différences des tradi- 
tions culturelles (le poids du puritanisme aux Etats-Unis), les différences dans les rapports hommes-femmes (plus grande fréquence des désunions aux Etats-Unis ; sociabilité entre les sexes moins crispée et plus d'intérêt érotique porté au plaisir de la femme en France), dans la fréquence des rapports sexuels (nettement plus élevée en France qu'aux Etats-Unis chez les 30 ans et plus), dans la palette des pratiques déjà essayées (plus riche en France : le cunnilingus et la sodomie hétérosexuelle, notamment, y sont plus répandus). Les résultats de l'enquête américaine et de l'enquête ACSF attestent ces différences.

Il faudrait, encore, constater que la société américaine est une société globalement plus “pressée " que la société française (cf. la devise puritaine : “ le temps c'est de l'argent”) et que cela n'est probablement pas sans effet sur la fréquence des rapports sexuels trop " pressés ". Bref, la France ne gagnerait rien à s'américaniser en matière d'érotisme et de relations entre les sexes, pas plus qu'en matière culinaire, par exemple.

Peut-on comparer la prévalence de l'EP, en France, en 1991-1992, à celle d'époques antérieures? Il n'y a pas de question sur l'EP dans l'enquête de P.Simon et al. [14]. Il faut donc recourir à un subterfuge.

Certains auteurs ont défini l'EP [16] comme une éjaculation se produisant en moins d'une minute (après la pénétration), en moins de 2 minutes, de 4 minutes (proposition de LoPiccolo en 1978), de 5 minutes ou de 7 minutes. Ces critères sont, bien sûr, arbitraires.

En 1970, 1\% des hommes de 20 ans et plus avaient déclaré un temps de pénétration moyen de 1 minute ou moins, $5 \%$ de 2 minutes, $6 \%$ de 3 minutes, $2 \%$ de 4 minutes, $17 \%$ de 5 ou 6 minutes. Cela correspondrait à des prévalences d'EP allant de $1 \%$ à $31 \%$. Les propositions intermédiaires paraissant plus réalistes, cela donnerait une prévalence d'EP persistante, en 1970 , allant de $12 \%$ à $14 \%$. Je n'en tirerai pas la conclusion que la prévalence de l'EP persistante a diminué entre 1970 et 1992 mais je crois, du moins, pouvoir en inférer qu'elle n'a probablement pas augmenté, alors que certains affirment parfois, sans preuve, que le féminisme des années 70, la prise en charge, par les femmes, de la responsabilité de la contraception, auraient favorisé une recrudescence des troubles sexuels masculins. Comme ni l'enquête américaine [10], ni l'enquête finlandaise [9] ne permettent des comparaisons sur ce point, nous devrons nous contenter de ces supputations en attendant une prochaine grande enquête sur la sexualité au XXIème siècle.

Signalons, à ce propos, que le temps consacré, en moyenne, aux préliminaires a dû, lui, augmenter un peu. En 1970 , le rapport sexuel durait en moyenne 26 minutes (31 minutes pour les hommes de 20-29 ans, 26 minutes pour ceux de 30-49 ans, 20 minutes pour ceux de 50 ans et plus). Dans chacune de ces trois tranches d'âge, le temps consacré aux préliminaires était égal au temps de pénétration (en moyenne donc, 13 minutes et 13 minutes). Or, la durée moyenne des rapports sexuels indiquée par les hommes, d'après l'enquête ACSF, a augmenté de 5 minutes en 22 ans (mais de 13 minutes pour les 20-29 ans). On peut supposer que ce gain a profité essentiellement aux préliminaires. Dans cette hypothèse, ceux-ci représenteraient aujourd'hui, en moyenne, près de $60 \%$ de la durée totale des rapports sexuels (18 minutes de préliminaires et 13 minutes de pénétration).

\section{LE CUMUL DE L'EJACULATION PREMATUREE ET DE LA DYSFONC- TION ERECTILE}

Les diverses dysfonctions sexuelles constituent, souvent, les unes pour les autres, des facteurs de risque curieusement négligés dans la plupart des analyses épidémiologiques les concernant, beaucoup moins bien étudiés, notamment, que les maladies cardio-vasculaires, l'hypertension, le diabète ou la consommation de tabac, d'alcool ou de médicaments, sur lesquels met l'accent, par exemple, l'étude MMAS consacrée à la DE [6].

Il convient de mettre en lumière par quelles transitions on passe de l'absence de dysfonctions au cumul occasionnel de plusieurs d'entre elles puis, éventuellement, à une situation où prédomine l'une d'entre elles, devenue persistante. 
Certes, l'enquête ACSF est une enquête transversale, fournissant un état des lieux à un moment donné (1991-1992). Ne pouvant la comparer à aucune autre enquête antérieure portant sur un échantillon représentatif de la population française (puisqu'elle est la première de ce genre à avoir tenté d'estimer la prévalence des dysfonctions sexuelles), nous ne pouvons être sûrs que certains effets liés à l'âge résultent seulement de l'âge et non de l'appartenance à une "génération " et donc de l'évolution culturelle qui favoriserait ou non la manifestation de telle ou telle dysfonction. La comparaison avec les autres enquêtes des années 90 (dans d'autres milieux culturels) montrant des similitudes dans les effets de l'âge sur la prévalence de l'EP et de la $\mathrm{DE}$, nous ferons l'hypothèse que la plupart de ces effets sont dûs au vieillissement et donc, par exemple, que la cohorte des hommes de 40 ans en 1992 a connu à peu près, depuis l'adolescence, en prenant de l'âge, les vicissitudes retracées dans la figure 1 pour les tranches 1824 et 25-39 ans. Pour l'établir de manière certaine, il aurait fallu, bien sûr, pouvoir réaliser des études longitudinales coûteuses ou encore accumuler des biographies sexuelles minutieuses, lesquelles poseraient des problèmes de mémorisation considérables, pas en ce qui concerne l'apparition brutale d'une DE persistante et irréversible (on ne l'oublie pas) mais certainement pour les troubles mineurs occasionnels qui " préparent" souvent les dysfonctions plus graves.

Je me concentrerai ici sur les cumuls entre l'EP - la dysfonction masculine la plus répandue ( $65 \%$ dont $11 \%$ souvent) - et la DE, laquelle est, à la fois, assez largement répandue ( $47 \%$ dont $7 \%$ souvent) et particulièrement redoutée. Je ne prendrai en considération, dans ce qui suit, que les hommes ayant eu au moins un rapport sexuel au cours des 12 mois précédant l'enquête ACSF, écartant ainsi les " abstinents 12 derniers mois " ce qui, du reste, modifie très peu les chiffres globaux. Les tranches retenues (cf. figure 1) seront dorénavant différentes de celles du tableau 1, afin, notamment, de pouvoir comparer les quadragénaires, les quinquagénaires et les sexagénaires. Une dysfonction sera dite "isolée " lorsqu'elle ne cohabite pas, chez une personne donnée, avec l'autre dysfonction ici considérée.
Chez l'ensemble des hommes de 18 à 69 ans, l'EP affecte $72 \%$ de ceux qui souffrent au moins occasionnellement de DE contre 60\% de ceux qui déclarent ne pas souffrir de $\mathrm{DE}$, et la $\mathrm{DE}$ affecte $52 \%$ des hommes souffrant au moins occasionnellement d'EP contre 39\% de ceux qui déclarent ne pas souffrir d'EP. On voit, ainsi, que l'EP au moins occasionnelle et la DE au moins occasionnelle sont statistiquement associées. Il en est de même pour l'EP " souvent " et la DE "souvent" : si le pourcentage d'EP " souvent" est de $11 \%$ chez tous, il monte à $32 \%$ chez ceux qui souffrent "souvent" de DE; le pourcentage de DE " souvent" est de $7 \%$ chez tous, de $21 \%$ chez ceux qui déclarent avoir "souvent" des EP.

Une manière simple d'apprécier si l'association entre ces deux dysfonctions est forte ou faible, est de comparer, dans la figure 1, le produit des pourcentages d'hommes cumulants et d'hommes n'ayant ni l'une ni l'autre, au produit des pourcentages d'hommes ayant l'EP isolée d'une part, la DE isolée d'autre part : si le premier produit (entre pourcentages de “ concordants") est nettement supérieur au second (entre pourcentages de "discordants") l'association est forte.

On voit immédiatement que l'association est assez faible à 18-24 ans, plus forte de 25 à 49 ans et qu'à partir de 50 ans elle devient négative, c'est-à-dire qu'on risque plus de souffrir d'une dysfonction si on n'a pas l'autre que si on a l'autre. Or, après 50 ans, plus d'hommes cumulent les deux dysfonctions que dans des tranches où celles-ci sont pourtant plus " associées" (par exemple chez les 25-39 ans). On en comprend la raison : à partir de 50 ans, on observe un bond des pourcentages d'hommes présentant soit l'EP isolée (bond chez les 50-59 ans) soit la DE isolée (bond chez les 60-69 ans) alors que les pourcentages de cumulants demeurent assez stables et que les pourcentages de " ni EP ni DE" diminuent.

Ainsi, l'évolution des rapports entre EP et DE selon l'âge se dessine de façon plus claire et plus concrète si l'on prête attention aux pourcentages de cumulants et de non cumulants dans la population des hommes ayant répondu à l'enquête (figure 1 et tableau 2), plutôt qu'à l'association statistique dans la "population des dysfonctions ".Considérons d'abord le 


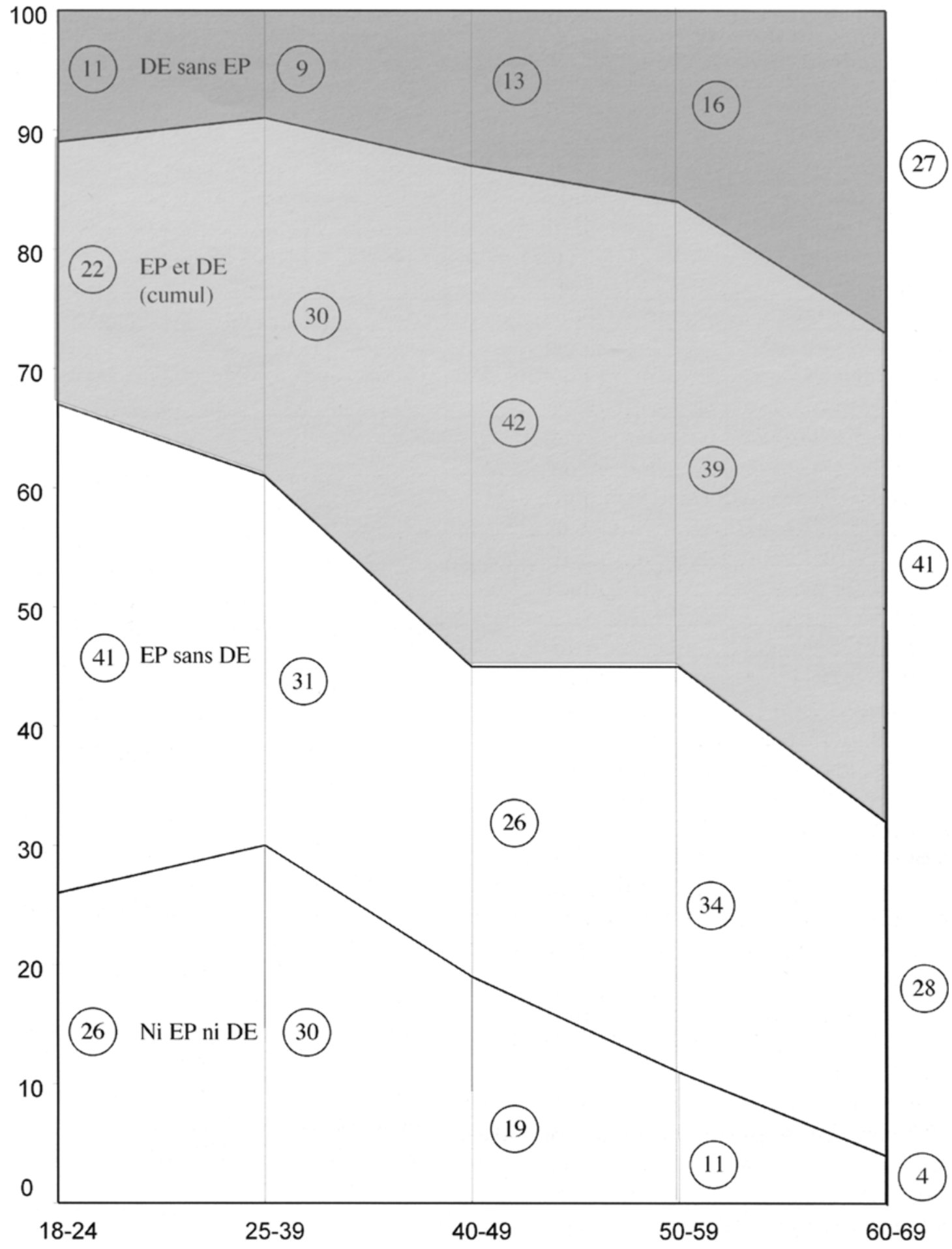

Figure 1: Chez les hommes ayant eu au moins un rapport sexuel dans les 12 mois précédant l'enquête ACSF (septembre 1991 - février 1992), pourcentages, par tranche d'âge, de ceux qui n'ont ni EP ni DE, qui ont au moins occasionnellement (souvent ou parfois ou assez rarement) l'une des deux dysfonctions mais pas l'autre, ou qui cumulent les deux. Lire : 33\% des hommes de 1824 ans ont déclaré souffrir au moins occasionnellement de DE (11\% de DE mais pas d'EP + $22 \%$ à la fois de DE et d'EP) N.B : les surfaces ne sont pas ici proportionnelles aux nombres d'hommes souffrant ou non de ces dysfonctions. 
Tableau 2 : Distribution du cumul (ou non-cumul) de l'éjaculation prématurée ante portas et/ou non ante portas (EP) et de la dysfonction érectile (DE) chez les hommes adultes vivant en France et ayant eu, à la date de l'enquête ACSF (septembre 1991-février 1992) au moins un rapport sexuel dans les 12 mois précédents, selon l'âge et les fréquences déclarées des deux dysfonctions (en \%)

\begin{tabular}{|l|c|c|c|c|c|c|}
\hline \multicolumn{1}{|c|}{ Age } & & & & & & \\
\hline $\begin{array}{l}\text { Fréquences déclarées des } \\
\text { deux dysfonctions }\end{array}$ & $18-24$ & $25-39$ & $40-49$ & $50-59$ & $60-69$ & $\begin{array}{c}\text { Ensemble } \\
18-69\end{array}$ \\
\hline EP jamais DE jamais & 26 & 30 & 19 & 11 & 4 & 21 \\
\hline $\begin{array}{l}\text { EP jamais DE rare } \\
\text { EP rare DE jamais } \\
\text { EP rare DE rare }\end{array}$ & 32 & 31 & 29 & 35 & 28 & 31 \\
\hline $\begin{array}{l}\text { EP jamais DE parfois } \\
\text { EP rare DE parfois }\end{array}$ & 4 & 3 & 6 & 7 & 8 & 5 \\
\hline $\begin{array}{l}\text { EP parfois DE jamais } \\
\text { EP parfois DE rare }\end{array}$ & 23 & 18 & 23 & 17 & 30 & 21 \\
\hline EP parfois DE parfois & 3 & 4 & 7 & 7 & 12 & 6 \\
\hline EP svt DE jamais & 6 & 4 & 3 & 12 & 4 & 5 \\
\hline EPsvt DE rare & 1 & 4 & 2 & 3 & 1 & 3 \\
\hline EP svt DE parfois & 0 & 1 & 1 & 0 & 2 & 1 \\
\hline EP jamais DE svt & 1 & 1 & 2 & 0 & 6 & 2 \\
\hline EP rare DE svt & 1 & 0 & 1 & 2 & 0 & 1 \\
\hline EP parfois DE svt & 2 & 1 & 4 & 3 & 3 & 2 \\
\hline EP svt DE svt & 1 & 3 & 3 & 3 & 2 & 2 \\
\hline Effectifs & 302 & 575 & 214 & 89 & 62 & 1242 \\
\hline
\end{tabular}

- les non-réponses sont exclues (elles sont inférieures à 2\%)

- l'adjectif rare est ici l'abréviation de assez rarement

- le total de chaque colonne est égal à 100

- lire : " 26\% des 18-24 ans ont déclaré n'avoir jamais ni EP ni DE ". 
cumul ou non-cumul entre EP persistante et DE persistante. Les "EP souvent mais pas DE souvent" sont 9\% des $18-69$ ans (mais $15 \%$ des 50-59 ans). Les " DE souvent mais pas EP souvent" sont 5\% des 18-69 ans (mais 2\% des 2539 ans et 9\% des 60-69 ans). Quant à ceux qui cumulent l'EP persistante et la DE persistante, leur pourcentage ne varie guère selon l'âge : il est de $1 \%$ chez les $18-24$ ans, de $3 \%$ de 25 à 59 ans et de $2 \%$ chez les 60-69 ans.

Examinons maintenant le cumul ou non-cumul EP-DE, les deux au moins occasionnellement (cf. figure 1). Ceux qui ont déclaré n'avoir ni EP ni DE sont $21 \%$ des 18-69 ans (30\% des 25-39 ans, $4 \%$ seulement des 60-69 ans). Ceux qui souffrent d'EP isolée (sans DE) sont $31 \%$ des $18-69$ ans $(41 \%$ des $18-24$ ans, $26 \%$ des $40-49$ ans). Les cumulants EP-DE sont $34 \%$ des 18 69 ans (22\% des 18-24 ans, $42 \%$ des $40-49$ ans). Enfin, ceux qui déclarent souffrir de DE mais pas d'EP sont $14 \%$ des $18-69$ ans (9\% des 25-39 ans mais $27 \%$ des 60-69 ans).

La figure 1 le montre bien : ceux qui n'ont aucun des deux troubles et ceux qui ont l'EP isolée sont progressivement "écrasés" par le poids des cumulants et, aussi, surtout à partir de 50 ans, par le poids de ceux qui déclarent une DE isolée (deux petites exceptions : le sursaut de ceux qui n'ont aucune des deux dysfonctions à 25-39 ans et le bond de l'EP isolée chez les 50-59 ans).

On trouve souvent dans les écrits sexologiques l'affirmation que l'EP serait surtout un problème des jeunes hommes. Or, le tableau 1 nous a montré que ce n'était pas le cas. L'affirmation susdite comporte, néanmoins, une part de vérité. Si nous prenons en considération l'EP isolée seulement, on voit qu'effectivement elle est beaucoup plus répandue chez les 18-24 ans que dans les autres tranches d'âge. De plus, chez les 18-24 ans, on observe une forte disparité entre le pourcentage d'EP isolée $(41 \%)$ et celui de $\mathrm{DE}$ isolée (11\%) alors que, par exemple, chez les 60-69 ans, ces pourcentages sont presque égaux (28\% d'EP isolée, $27 \%$ de $\mathrm{DE}$ isolée). Ou encore, $55 \%$ des jeunes hommes de 18-24 ans ayant déclaré pâtir d'EP et/ou de $\mathrm{DE}$ ont déclaré souffrir d'EP isolée ; ce pourcentage tombe à $29 \%$ chez les 60-69 ans.
L'EP non accompagnée de DE affecte donc plus souvent les jeunes. On invoque habituellement, pour expliquer ce phénomène l'appréhension de ne pas réussir, "l'anxiété de la performance" (aux Etats-Unis, elle est effectivement un peu plus souvent déclarée par les 1824ans [10]), l'émotivité, l'inexpérience érotique, le manque de régularité de la vie sexuelle, les mauvaises conditions dans lesquelles s'accomplissent les rapports sexuels, l'habitude d'une masturbation trop rapide ou accompagnée de sentiments de culpabilité, des difficultés parfois à fantasmer, la crainte de la femme, génératrice d'agressivité à son égard et d'indifférence à son plaisir ou, à l'opposé, un désir excessif de fusion avec elle, suscitant la peur de lui faire mal.

Il n'est pas possible, à partir de l'enquête ACSF, de tester toutes ces hypothèses. Nos effectifs sont insuffisants pour étudier précisément, par exemple, les 18-24 ans souffrant d'EP isolée. Mais le tableau 3 apporte quelques informations intéressantes. Les 18-39 ans, quand ils souffrent d'EP persistante, ont un nombre médian de rapports sexuels sur 4 semaines légèrement supérieur à celui des autres hommes de la tranche d'âge et leur satisfaction sexuelle n'est pas affectée par la dysfonction.

Les jeunes hommes, en effet, parviennent souvent à bien " compenser ", c'est-à-dire à prolonger et à varier les préliminaires (en tirant, éventuellement, plaisir de la satisfaction de la partenaire) ou encore, profitant de ce que leur " période réfractaire" est encore courte, à recommencer un nouveau coït vaginal après une éjaculation trop rapide (en d'autres termes, à “ remettre le couvert"). Les partenaires de ces jeunes hommes insuffisamment expérimentées, ou pas encore désabusées, peuvent même parfois se sentir flattées de susciter un si vif désir. Mais tout ceci n'a qu'un temps. On voit, dans le tableau 3 , que chez les 40-69 ans, à la fois la fréquence des rapports et la satisfaction sont très affectées par la dysfonction (et vice versa).

Remarquons, par parenthèse, dans ce même tableau, que pour ceux qui échappent à l'EP (et aux autres dysfonctions avec lesquelles elle se cumule), l'âge (du moins jusqu'à 69 ans) ne 
Tableau 3 : Nombres médians de rapports sexuels au cours des 4 semaines précédant l'enquête ACSF (septembre 1991-février 1992) et pourcentages de "très satisfaits de leur vie sexuelle actuelle "chez les hommes adultes vivant en France et ayant eu au moins un rapport sexuel dans les 12 mois précédents, selon l'âge et la fréquence déclarée de leur éventuelle éjaculation prématurée (EP).

\begin{tabular}{|c|c|c|c|c|}
\hline \multicolumn{2}{|c|}{$\begin{array}{l}\text { Age et fréquence déclarée de } \\
\text { l'EP }\end{array}$} & $\begin{array}{l}\text { Nombres médians de } \\
\text { rapports } 4 \text { semaines }\end{array}$ & $\begin{array}{l}\text { Pourcentages de } \\
\text { " très satisfaits " }\end{array}$ & Effectifs \\
\hline \multirow{3}{*}{$\begin{array}{c}\text { Hommes } \\
\text { de }\end{array}$} & EP souvent & 6 & 43 & 76 \\
\hline & EP parfois & 5 & 40 & 209 \\
\hline & $\begin{array}{l}\overline{\mathrm{EP}} \text { assez } \\
\text { rarement }\end{array}$ & 5 & 43 & 238 \\
\hline $18-39$ ans & EP jamais & 5 & 45 & 354 \\
\hline \multirow{3}{*}{$\begin{array}{c}\text { Hommes } \\
\text { de }\end{array}$} & EP souvent & 4 & 35 & 38 \\
\hline & EP parfois & 5 & 32 & 109 \\
\hline & $\begin{array}{l}\overline{\mathrm{EP}} \text { assez } \\
\text { rarement }\end{array}$ & 5 & 45 & 83 \\
\hline $40-69$ ans & EP jamais & 8 & 49 & 135 \\
\hline
\end{tabular}

semble nuire ni à la fréquence des rapports ni à la satisfaction, bien au contraire. Le vieillissement, débarrassé de ses mauvais aspects, améliorerait-il (au moins jusqu'à 69 ans) la qualité de la vie sexuelle?

Bref, chez les jeunes hommes, l'EP et la DE, le plus souvent, ne cohabitent pas, et l'on peut observer de grandes différences entre le caractère et la biographie sexuelle des jeunes souffrant d'EP isolée et de ceux affligés de DE isolée.

A l'âge mûr, les dysfonctions cohabitent plus souvent chez les mêmes sujets : les transformations de l'une en l'autre, les renforcements réciproques se font plus fréquents. On pourrait dire qu'en cohabitant elles finissent par se ressembler de plus en plus.

Après 50 ans, quand les deux dysfonctions ne cohabitent plus chez les mêmes sujets, c'est souvent que l'une a pris, au fil des jours, la place de l'autre après avoir plus ou moins longtemps cohabité avec elle. Cela évoque le processus d" exclusion compétitive " entre espèces voisines occupant les mêmes niches écologiques et entrant, de ce fait, en une concurrence qui amène l'éviction, voire l'extinction, de l'une d'entre elles.
Souvent " complémentaires " dans les tranches d'âge précédentes, elles sont devenues " concurrentes " et, dans cette " concurrence", la DE isolée l'emporte de plus en plus fréquemment sur sa " rivale". Pourquoi ?

Tout d'abord, les facteurs organiques liés à l'âge favorisent plus la survenue de la $\mathrm{DE}$ isolée que celle de l'EP isolée. D'autre part, quand elle devient persistante, la DE peut supprimer l'envie, voir la possibilité, d'éjaculer. Souvent encore, les éjaculateurs trop rapides, pour retarder leur éjaculation, recourent à des moyens (par exemple, penser à des choses désagréables) qui, réduisant le désir, peuvent provoquer l'impuissance. En outre, chez certains hommes, surtout après 60 ans, l'EP peut s'effacer spontanément car l'âge leur permet de mieux se retenir : la place est ainsi libre pour une éventuelle DE isolée.

Il peut arriver cependant, mais plus rarement, que ce soit l'EP qui “ écarte " la DE. En s'escrimant à ne pas perdre une érection devenue instable, l'homme bouge trop (ce qui, on le sait, ne permet guère de retarder l'éjaculation) ou bien encore, il préfère éjaculer au plus vite, par peur de la panne, " choisissant" ainsi la dysfonction qui lui semble constituer le moindre mal. 


\section{UNE TYPOLOGIE DES EJACULA- TIONS PREMATUREES}

A partir de ces observations et de ces hypothèses, je propose de distinguer entre quatre types principaux d'EP : deux formes "isolées " (c'est-à-dire sans cumul), l'une majoritaire chez les jeunes hommes (type 1), l'autre affectant particulièrement les quinquagénaires (type 4); et deux formes avec cumul, le type 2 (se produisant surtout à l'âge mûr, entre 25 et 50 ans), et le type 3 (surtout après 50 ans).

\section{L'EP de type 1}

D'apparition précoce (souvent " primaire "), généralement bien compensée, non accompagnée de DE, elle constitue la forme d'EP majoritaire avant 25 ans. Cette EP “ juvénile " n'est pas, pour ceux qui la déclarent, généralement associée à l'image d'un manque de virilité (surtout si le modèle de la répétition des coïts brefs est culturellement valorisé). Elle ne retentit ni sur la fréquence des rapports ni sur la satisfaction (cf. tableau 3), même si au bout d'un temps elle nuit à l'entente affective et sexuelle du couple. Elle s'oppose, de ce point de vue, à la $\mathrm{DE}$ " juvénile " primaire psychogène, laquelle, si elle ne bénéficie pas d'une rapide rémission spontanée, a généralement des effets importants sur l'entente du couple, la fréquence des rapports, voire sur l'orientation sexuelle de celui qui en souffre. Il semble, d'après certains cliniciens, que la plupart des hommes qui consultent spécifiquement pour une EP, se rattachent à ce type. En effet, les hommes plus âgés qui présentent les deux troubles à la fois affirment être plutôt préoccupés par celui des deux problèmes qui leur paraît le plus grave, la DE.

\section{L'EP de type 2}

Cette EP accompagnée de DE est la forme d'EP majoritaire entre 25 et 50 ans, c'est-à-dire dans les tranches d'âge bénéficiant de l'activité sexuelle la plus régulière. Cette EP de l'âge mûr est souvent moins bien compensée et, au fil des jours, elle s'accompagne, de plus en plus souvent, de DE. Dans certains cas, l'expérience occasionnelle d'une instabilité de l'érection provoque la peur d'avoir une impuissance complète (une " peur de l'échec "), laquelle entraî- ne une éjaculation plus rapide afin de ne pas perdre l'érection : l'apparition du trouble de l'érection a donc ainsi contribué à aggraver une EP qui lui préexistait. Dans d'autres cas, c'est l'aggravation de l'EP (devenue plus souvent persistante et se produisant plus fréquemment ante portas cf. tableau 1) qui favorise l'apparition ou l'aggravation de la DE. Dans tous ces cas, la partenaire voit de plus en plus mal ce qui distingue, quant à l'effet sur son propre plaisir, une EP, surtout ante portas, persistante, d'une impuissance. Elle le fait éventuellement sentir à l'homme, ce qui peut faire perdre à celui-ci l'illusion qu'une EP même parfaitement compensée serait un signe de virilité, ce qui peut, de surcroît, provoquer ou renforcer chez lui l'anxiété de la performance. Remarquons également qu'un homme sur deux souffrant de cette EP de type 2 déclare avoir au moins occasionnellement des éjaculations retardées, ce qui pourrait sembler un paradoxe mais résulte probablement des difficultés croissantes de la compensation (ou de l'évitement de l'impuissance) et de la perte relative de désir qui s'ensuit.

\section{L'EP de type 3}

Cette EP (accompagnée de DE) de l'homme vieillissant est la forme d'EP majoritaire après 50 ans. Elle peut être favorisée par une détérioration générale de l'état de santé, par une $\mathrm{DE}$ organique d'apparition récente (et souvent soudaine), par des soucis professionnels et familiaux. Mais elle est plus souvent encore liée à une détérioration de la vie sexuelle et affective du couple. Les rapports sexuels s'espacent, ce qui, à cet âge, favorise la manifestation de l'EP (cf. tableau 3). La femme (parfois, en outre, confrontée aux difficultés de la ménopause) ne supporte plus d'être privée de plaisir et participe de moins en moins activement à la stimulation physique de son partenaire, à un moment où cette participation est pourtant indispensable pour l'homme (lui-même moins stimulé psychologiquement) : tout ceci favorise la DE ou entraîne, pour le moins, des érections capricieuses ou instables. Cette forme d'EP accompagnée de DE peut se manifester par des "éjaculations sur verge molle", que la partenaire peut éventuellement provoquer par des caresses ou par la fellation. Elle finit parfois 
mais non nécessairement par se transformer en une DE pure et simple, en particulier lorsqu'elle ne peut plus être compensée.

\section{L'EP de type 4}

Cette EP isolée est une forme minoritaire d'EP de l'homme vieillissant. Elle semble affecter particulièrement les quinquagénaires (voir le tableau 2 : $3 \%$ des hommes de $40-49$ ans souffrent d'EP persistante isolée ; ce pourcentage chez les hommes de 50-59 ans monte à $12 \%$ ). Elle peut être parfois primaire. Mais elle constitue probablement, pour la plupart de ceux qui en souffrent, une transition entre l'EP de type 2 et l'EP de type 3 . Elle semble, en effet, procéder souvent d'une accélération de l'éjaculation visant à profiter de ses bonnes dispositions afin d'échapper à une dysfonction jugée plus grave, l'impuissance. Elle traduit ainsi une perte de confiance en ses capacités qui risque de s'accentuer au fil des ans.

Notons, pour terminer, que la fréquence des cumuls EP-DE a deux conséquences qu'il convient de souligner :

- le traitement (pharmacologique ou autre) spécifique d'une des dysfonctions cumulées risque souvent de laisser subsister l'autre ou les autres dysfonctions sexuelles dont souffrent également les patients.

- les interactions de l'EP avec la DE organique et/ou psychogène compliquent considérablement la tâche des thérapeutes des dysfonctions sexuelles : elles pourraient expliquer, en partie, les résultats assez médiocres -auxquels j'ai fait allusion au début de l'article - des thérapies comportementalistes actuelles de l'éjaculation prématurée.

Remerciements à : Patrick de Colomby et François Giuliano pour de stimulants échanges d'idées à l'occasion d'un travail commun sur l'épidémiologie de la DE ; Madame Fugier qui a réalisé la saisie informatique du présent article ; Monsieur J.C.Czyba pour ses encouragements et sa patience.

\section{REFERENCES}

1. ACSF INVESTIGATORS : AIDS and sexual behaviour in France. Nature, 1992, 360,407-409.

2. ANONYME : L'art priapique (1864). In : L'érotisme Second Empire (Pauvert J.-J. ed.) Paris, Carrere, $1985,382 \mathrm{p}$.

3. BÉJIN A. : Le nouveau tempérament sexuel. Essai sur la rationalisation et la démocratisation de la sexualité. Paris, Editions Kimé, 1990, 136p.

4. BÉJIN A. : Plaisirs sexuels, dysfonctions, fantasmes, satisfaction. In : Spira A., Bajos N. et le groupe ACSF. Les comportements sexuels en France. Paris, La Documentation Française, 1993, 194-202.

5. BÉJIN A. L'orgasme de l'homme adulte. Quelques résultats des études quantitatives récentes sur le comportement sexuel, notamment en France. Andrologie, 1997, 7, ${ }^{\circ} 3,336-342$.

6. FELDMAN H.A., GOLDSTEIN I., HATZICHRISTOU D.G., KRANE R.J., Mc KINLAY J.B. : Impotence and its medical and psychosocial correlates : Results of the Massachusetts Male Aging Study. J.Urol., Vol. 151, January 1994, 54-61.

7. KINSEY A.C., POMEROY W.B., MARTIN C.E. : Sexual behavior in the human male. Philadelphia, Saunders, 1948, XVI- 804 p.

8. KINSEY A.C., et al. Sexual behavior in the human female. Philadelphia, Saunders, 1953, XXX- 842 p.

9. KONTULA O., HAAVIO-MANNILA E. : Sexual pleasures. Enhancement of sex life in Finland 1971-1992. Aldershot, Dartmouth, 1995, X-287p.

10. LAUMANN E.O, GAGNON J.H, MICHAEL R.T., MICHAELS S. : The social organization of sexuality. Sexual practices in the United States. Chicago. The University of Chicago Press, 1994, XXXII-718 p.

11. MASTERS W.H., JOHNSON V.E, KOLODNY R.C. Amour et sexualité (1986). Paris, Inter Editions, 1987, $588 \mathrm{p}$.

12. METZ M.E, PRYOR J.L., NESVACIL L.J., FARUK ABUZZAHAB S.R., KOZNAR J. : Premature ejaculation : A psychophysiological review. Journal of Sex and Marital Therapy, 1997, 23, $\mathrm{n}^{\circ} 1,3-23$.

13. OVIDE L'art d'aimer. Livre II (1 av. J.C.) trad H.Bornecque. Paris, Livre de Poche, 1970, 191 p.

14. SIMON P., GONDONNEAU J., MIRONER L., DOURLEN-ROLLIER A.M. : Rapport sur le comportement sexuel des Français. Paris, R.Julliard et P.Charron, 1972, 922p.

15. SPIRA A., BAJOS N., et LE GROUPE ACSF : Les comportements sexuels en France. Paris. La Documentation Française, 1993, 352 p.

16. TRUDEL G., CARUFEL F., FORTIER P., LANDRY L. : L'éjaculation précoce : une recension des écrits. 
Revue sexologique (Montréal), 1996, 4, n², 7-34.

17. VÂTSYÂYANA, Kâma Sûtra (4ème siècle ap. J.C.), trad. A.Danielou. Paris, Editions du Rocher, 1992, $623 p$.

\begin{abstract}
The epidemiology of premature ejaculation and of its association with erectile dysfunction
\end{abstract}

\begin{abstract}
A. BÉJIN
A massive survey of sexual lifestyles has been conducted in France (Nature 1992, 360, 407409 ) in response to the spread of the AIDS epidemic. This survey was applied to 20055 people aged 18-69 years and was focused on a detailed description of sexual practices and means of protection against AIDS contamination. Questions concerning sexual dysfunctions were included in an additional questionnaire. This article examines the most common male sexual dysfunction, premature ejaculation (PE) and its association with erectile dysfunction (ED). The prevalence rate of PE reported "often" was 11\% (ED reported "often" : 7\%). The prevalence rate of $\mathrm{PE}$ reported "often" or "sometimes" or "quite seldom" was $65 \%$ (ED : 47\%). With regard to the association of $P E$ with $E D, 26 \%$ of men aged 18.24 reported neither $P E$ nor $E D, 11 \%$ reported only ED, $41 \%$ only PE, and $22 \%$ both PE and $\mathrm{ED} ; 4 \%$ of men aged $60-69$ reported neither $\mathrm{PE}$ nor ED, $27 \%$ reported only ED, $28 \%$ only PE, and $41 \%$ both PE and ED. I discuss these prevalence rates and $I$ propose a typology of PE.
\end{abstract}

Key-Words : Sexuality - epidemiology - premature ejaculation - erectile dysfunction - sexual desire France - international comparisons. 